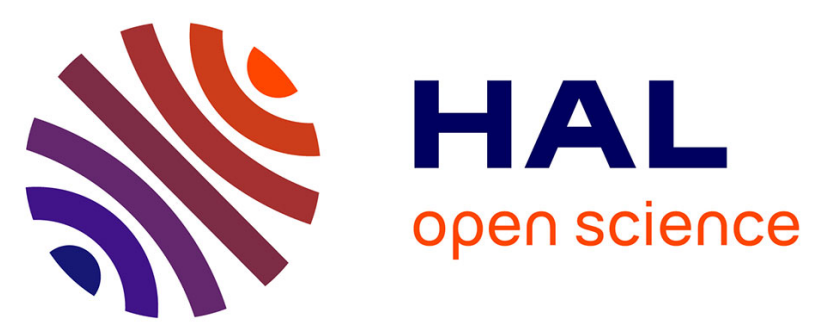

\title{
Unravelling molecular mechanisms in the fluorescence spectra of doxorubicin in aqueous solution by femtosecond fluorescence spectroscopy
}

P. Changenet-Barret, T. Gustavsson, D. Markovitsi, I. Manet, S. Monti

\section{- To cite this version:}

P. Changenet-Barret, T. Gustavsson, D. Markovitsi, I. Manet, S. Monti. Unravelling molecular mechanisms in the fluorescence spectra of doxorubicin in aqueous solution by femtosecond fluorescence spectroscopy. Physical Chemistry Chemical Physics, 2013, 15 (8), pp.2937-2944. 10.1039/C2CP44056C . hal-00807306

\section{HAL Id: hal-00807306 https://hal.science/hal-00807306}

Submitted on 3 Apr 2013

HAL is a multi-disciplinary open access archive for the deposit and dissemination of scientific research documents, whether they are published or not. The documents may come from teaching and research institutions in France or abroad, or from public or private research centers.
L'archive ouverte pluridisciplinaire HAL, est destinée au dépôt et à la diffusion de documents scientifiques de niveau recherche, publiés ou non, émanant des établissements d'enseignement et de recherche français ou étrangers, des laboratoires publics ou privés. 


\title{
Unravelling molecular mechanisms in the fluorescence spectra of Doxorubicin in aqueous solution by femtosecond fluorescence spectroscopy
}

\author{
Pascale Changenet-Barret, ${ }^{a}$ Thomas Gustavsson, ${ }^{a}$ Dimitra Markovitsi, ${ }^{a}$ Ilse Manet, ${ }^{* b}$ and Sandra Monti ${ }^{b}$ \\ ${ }_{5}$ Received (in $\left.X X X, X X X\right) X$ th $X X X X X X X X X 20 X X$, Accepted $X$ th $X X X X X X X X X 20 X X$ \\ DOI: 10.1039/b000000x
}

Doxorubicin (DOX) is a potent anti-tumoral agentwidely used for cancer therapy. Despite numerous studies, the fluorescence properties of DOX, usually exploited for the characterization ofthe interaction with biological media,have until now led to controversial interpretations, mainly due to self-association of

10 the drug in aqueous solution. We present here thefirst femtosecond study of DOX based on measurements with fluorescence up-conversion technique in combination with time-correlated single photon counting using the same laser source. We provide evidence that fluorescence signals of DOX stem from monomers and dimers. DOX dimerization induces a dramatic decrease in the fluorescence quantum yield from $3.9 \times 10^{-2}$ to $10^{-5}$ associated to the red shift of the fluorescence spectrum by $c a \sim 25 \mathrm{~nm}$. While the

15 fluorescence lifetime of themonomer is $1 \mathrm{~ns}$, the dimer fluorescence is found to decay with a lifetime of about 2 ps.In contrast to monomers, the fluorescence anisotropy of dimers is found to be negative. These experimental observations are consistent with an ultrafastinternal conversion $(<200 \mathrm{fs})$ between two exciton states, possibly followed by a charge separation process.

\section{${ }_{20}$ 1. Introduction}

Anthracycline antibiotics are very efficient anti-tumoral agents extensively employed in chemotherapy for the treatment of a large variety of cancers. Although the exact mechanism of their action is still not clear, theyare thought to act as inhibitors of both ${ }_{25}$ DNA replication and RNA transcription. It is generally accepted that this biological activity relies on their interactions with BDNA structures, ${ }^{1-3}$ through the intercalation of the anthraquinone moiety at the GC sequences, while the drug sugar moiety interacts with the minor DNA groove. ${ }^{4,}{ }^{5}$ Doxorubicin (DOX), 30 also known as Adriamycin (Fig. 1), is a natural anthracyclinecompound which is considered to be a prototypical anti-tumordrug. ${ }^{6-9} \mathrm{DOX}$ consists of a tetrahydroxyanthraquinonechromophore with a pendant glycosyl moiety, representing the essential structural features of anthracyclines.

35 The anthraquinonechromophore contains two hydroxyl groups respectively positioned in direct neighbourhood of the central oxo group.

Owing to its great efficacy, DOX is still widely used in clinical practice, in spite of the deleterious side effects mainly related to 40 its cardiotoxicity. More recently, another serious problem in DOX treatment has emerged connected to the development of some resistant cancer cell lines. ${ }^{6,7}$ In the frame of its clinical use, the poor solubilityof DOX in aqueous solution is considered to be a serious drawback, affectingboth its transport across the cell
45 membranes and its biological activity. ${ }^{10}$ In this respect, the development of nano-sized drug carriersfor DOX has recently attracted a lot of research interest, since it may offer the right solutions to overcome the problems related to cytotoxicity, solubility and resistance. ${ }^{11-19}$

50

Fig. 1 Doxorubicin $(\mathrm{DOX})$

In view of the design of innovative biomedical applications involving DOX, understanding the nature of the interactions of DOX with its environment,once dissolved in aqueous solution,is 55 of paramount importance. In this respect, the physical properties of DOX in aqueous solution have been the object of a large variety of studies including UV-vis absorption, NMR, circular dichroism (CD) and fluorescence spectroscopy. ${ }^{20-28}$ While concentration dependent absorption, NMR and CD spectra are ${ }_{60}$ generally interpreted in terms ofdimer formation, there is only a limited and controversialunderstanding of the intrinsic fluorescence properties of the drug in solution. Aggregation is 
known to induce a significant decrease of the fluorescence quantum yield attributed to the formation of non-fluorescent dimers. ${ }^{23,}{ }^{27}$ Fluorescence of DOX in aqueous solution has been reported alternatively to exhibit monoexponential or 5 biexponential decays. ${ }^{24}$, ${ }^{28-33}$ The origin of such biexponentialdecays is not fully clarified and has led to various interpretations. The presence of two ground-state tautomers or two ground-state conformers differing in their inter-molecular hydrogen bonds with the solvent, as well as the formation of 10 astrongly fluorescent photodegradation producthave been proposed. ${ }^{24,} 32-35$ These contrasting interpretations prompted us to examine the intrinsic photophysics of DOXin aqueous solution prior to studying the interaction of the drug with biological species or nano-sized particles. The aimofthis work is to 15 disentanglethe various molecular contributions to the fluorescence signals taking also into consideration the aggregation tendency of DOX. To this purpose, a combination of femto- and picosecond fluorescence techniques was used. The fluorescence decays, fluorescence anisotropy decays at selected 20 wavelengths and time-resolved spectra have been measured for different concentrations of DOX, in aqueous solutions, at physiological conditions. The overall results are discussed in the frame of the literature on anthracyclines and their close derivatives.

\section{${ }_{25}$ 2. Materials and Methods}

\subsection{Materials}

DOX (purity >95\%)was purchased from ALEXIS Biochemicals and used without any further purification. Solutions of DOX were prepared in ultra-pure water delivered by a Millipore MilliQ 30 system. Aqueous solutions of DOX at physiological conditions were prepared in $10 \mathrm{mM}$ Tris buffer, at $\mathrm{pH} 7.4$, with $1 \mathrm{mM}$ EDTA and $50 \mathrm{mM} \mathrm{NaCl}$. For the time resolved-fluorescence measurements, concentration of DOX was varied between $10 \mu \mathrm{M}$ and $1 \mathrm{mM}$.

\section{2.2. Steady-statespectroscopy}

Steady-state absorption and fluorescence spectra were recorded with a double-beam UV-visible spectrophotometer (Perkin-Elmer Lambda 900) and spectrofluorimeter (SPEX Fluorolog-3). For steady-state fluorescence measurements, $1 \mathrm{~cm}$ thickness quartz 40 cells were used. The fluorescence spectra were corrected for the wavelength-dependent response of the instrument.

\subsection{Time-resolved fluorescence measurements}

Time-resolved fluorescence measurements were carried out with two different techniques: time-correlated single photon counting 45 (TCSPC) and fluorescence up-conversion (FU) described in detail elsewhere. ${ }^{36,37}$ In both cases, the laser source was a modelocked Ti-sapphire laser providing 120 -fs pulses at $800 \mathrm{~nm}$ with a repetition rate of $76 \mathrm{MHz}$ (MIRA, Coherent). For excitation, frequency-doubled pulses at $400 \mathrm{~nm}$ were used.For the TCSPC 50 measurements, the laser repetition rate was reduced to $4.75 \mathrm{MHz}$ using a pulse-picker (Coherent) and the average excitation power was set to less than $1 \mathrm{~mW}$. The polarization of the exciting beam was controlled with a zero-order half-wave plate. For the FU measurements, the average excitation power was set to $60 \mathrm{~mW}$. 55 The fluorescence from the sample was collected with parabolic mirrors and mixed with the residual fundamental in a $0.5 \mathrm{~mm}$ type I BBO crystal to generate the sum-frequency light. The latter was spectrally filtered in a monochromator and detected by a photomultiplier in single-photon counting mode. The spectral 60 resolution was approximately5 $\mathrm{nm}$. Time-resolved fluorescence spectra in the 500-700 $\mathrm{nm}$ region were recorded at magic angle, by spectral scans on three different time scale, with time steps of $100 \mathrm{fs}, 1 \mathrm{ps}$ and $10 \mathrm{ps}$ respectively. The acquisition procedure of these spectra has been addressed in details in a previous report. ${ }^{38}$ ${ }_{65}$ Note that the different spectral components of the up-converted signal travel at different speeds through the various optics due to the group velocity dispersion. Therefore,the delay stage was adjusted in order to compensate the wavelength-dependent difference in group velocity, during the spectral scans. The time70 integrated spectra were corrected a posteriorifor the spectral response of the detection, with regards to the steady-state fluorescence spectrum.

Both for TCSPC and FU, fluorescence decays were measured with parallel and perpendicular excitation-detection polarization 75 configurations by adjusting the polarization of the excitation beam with a zero-order half-wave plate.

All time-resolved fluorescence measurements were performed at room temperature $\left(20 \pm 1^{\circ} \mathrm{C}\right)$, under aerated conditions. For TCSPC, the sample solution was placed in a $1 \mathrm{~cm}$ thick quartz 80 cell and magnetically stirred.For FU, $1 \mathrm{~mm}$ or $2 \mathrm{~mm}$ thick quartz cells were used. The cell was kept rotating during acquisitions. Absorption and fluorescence spectra of the sample were checked before and after experiment.

\subsection{Data analysis}

85 Total fluorescence kinetics $\mathrm{F}(\mathrm{t})$ were constructed from $\mathrm{I}_{\text {par }}(\mathrm{t})+2 \times \mathrm{G} \times \mathrm{I}_{\text {perp }}(\mathrm{t})$, fluorescence anisotropy decays $\mathrm{r}(\mathrm{t})$ were calculated according to $\left(\mathrm{I}_{\text {par }}(\mathrm{t})-\mathrm{G} \times \mathrm{I}_{\text {perp }}(\mathrm{t})\right) / \mathrm{F}(\mathrm{t})$. $\mathrm{G}$ is the ratio of the sensitivity of the detection system to parallel (vertical) and perpendicularly (vertical) polarized excitation light. In our case $G$ 90 was measured to be 1.0 for both fluorescence set-up.

Total fluorescence kinetics were independently fitted with a sum of exponential functions, convoluted by a Gaussian function representing the instrument response function. The full width at half maximum (FWHM) of the Gaussian was found to be about ${ }_{95} 280 \pm 15$ fs at all probed wavelengths.

The rotational diffusion time $\tau_{\text {Rot }}$ and the fluorescence anisotropy $r_{0}$ were extracted from the global fit of $I_{\text {par }}(t)$ and $I_{\text {perp }}(t)$ with convolution fitting routines, following equations:

$$
\begin{gathered}
\mathrm{I}_{\mathrm{par}}(\mathrm{t})=(1+2 * r(\mathrm{t})) * \mathrm{f}(\mathrm{t}) \\
\mathrm{I}_{\text {perp }}=(1-\mathrm{r}(\mathrm{t})) * \mathrm{f}(\mathrm{t}) \\
\text { withr }(\mathrm{t})=\sum_{\mathrm{i}=1}^{\mathrm{m}} \mathrm{r}_{\mathrm{i}} \exp \left(-\mathrm{t} / \tau_{\mathrm{i} \text { Rot }}\right), \\
\operatorname{andf}(\mathrm{t})=\sum_{\mathrm{j}=1}^{\mathrm{n}} \mathrm{a}_{\mathrm{j}}{ }^{*} \exp \left(-\mathrm{t} / \tau_{\mathrm{j}}\right)
\end{gathered}
$$

The full set of kinetic profiles extracted from the time-resolved fluorescence spectra were globally fitted, after dimensional reduction and noise filtering by singular value decomposition (SVD), ${ }^{39}$ as previously described. ${ }^{40}$ The results of the global 105 analysis are presented as Decay Associated Spectra (DAS) corresponding to the spectral distribution of the amplitudes associated to the time components. 


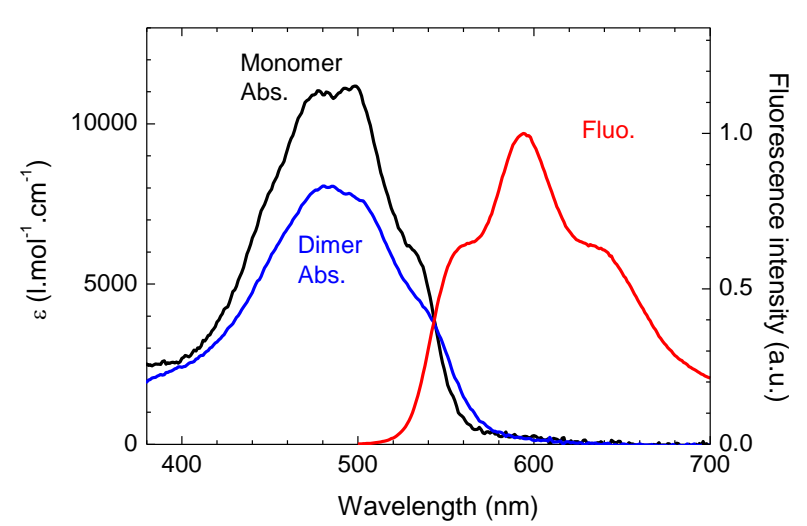

Fig. 2Black and blue lines are the absorption coefficients of DOX monomers and one DOX molecule in dimers, respectively, calculated assuming a dimerization binding model in the analysis of a series of spectra obtained for varying DOX concentration. ${ }^{28}$ In red, corrected steady-state fluorescence spectra $\left(\lambda_{\text {exc }}=350,400\right.$ or $\left.450 \mathrm{~nm}\right)$ for $10 \mu \mathrm{M}$ DOX solution in Tris buffer.

\section{Results and discussion}

\subsection{Steady-state spectroscopy of DOX and self-association}

${ }_{10}$ Fig. 2 illustrates the steady-state absorption and fluorescence spectra of a $10 \mu \mathrm{M}$ DOX solution in Tris buffer at $\mathrm{pH}$ 7.4. These spectra display similar features to those of 1,4-dihydroxy anthraquinone (quinizarin) in protic solvents. ${ }^{41}$ The absorption spectrum of DOX displays one main band centred at $490 \mathrm{~nm}$ 15 associated to the ${ }^{1} \mathrm{~A} \rightarrow{ }^{1} \mathrm{~L}_{\mathrm{b}} \pi, \pi *$ transition polarized along the long axis ${ }^{42}$ anda shoulder around $360 \mathrm{~nm}$ attributed to partially forbidden $\mathrm{n}, \pi^{*}$ transitions involving the three $\mathrm{C}=\mathrm{O}$ groups. ${ }^{22}$

The fluorescence spectrum of DOX exhibits three distinct peaks around 560, 594 and $638 \mathrm{~nm}$ associated to a vibrational 20 progression with a frequency lying in the range of 1,000$1,200 \mathrm{~cm}^{-1}{ }^{21}$ As in the case of quinizarin, the vibrational structures observed in the absorption and the fluorescence spectra stem fromthe symmetric modes associated with the $\mathrm{C}=\mathrm{O}$ bending, skeletal stretching and $\mathrm{OH}$ bending motions of the 25 dihydroxyanthraquinone moiety. ${ }^{21,43}$

The visible region of the absorption spectrum of DOX is known to be strongly influenced by the protonation state of the dihydroxyanthraquinone moiety whereasit is barely sensitive to the protonation state of the sugar part which is not conjugated to 30 the central aromatic ring. The $\mathrm{pK}_{\mathrm{a}}$ of the daunosamine- $\mathrm{NH}_{3}{ }^{+}$ group and one of the phenolic $\mathrm{OH}$ groups of the anthracyclinering $\mathrm{B}$ in aqueous solutionsbeing 8.15 and 10.16 , respectively. ${ }^{44}, 45$ Under physiological conditions, the phenol moietyremains thereforeneutral whereas the daunosamin- $\mathrm{NH}_{3}{ }^{+}$is expected to be 35 positively charged for a large fraction of molecules $(\sim 82 \%){ }^{22,42}$

Aggregation is also known to affect significantly the absorption spectrum of DOX leading to a slight broadening of the main absorption band with a significant hypochromism in the spectral region between $415 \mathrm{~nm}$ and $540 \mathrm{~nm}$ and a weak 40 hyperchromism in the spectral region above $540 \mathrm{~nm} .{ }^{20}$, ${ }^{46}$ Literature provides quite different values for the association constant of DOX in aqueous solutions, depending on the experimental conditions. ${ }^{20-22,26-28}$ For concentrations below $5 \mathrm{mM}$, a simple dimerization model is in general considered sufficient to
45 describe theself-association process. ${ }^{27}$ Under these conditions, a previous analysis of the absorption spectra of DOX in Tris buffer, at physiological $\mathrm{pH}$ yielded a dimerization constant with $\log \left(\mathrm{K}_{\mathrm{d}}\right)$ $=4.8 \pm 0.1 .{ }^{28}$ Using this value, it can be estimated that, a fraction of $47 \%$ of the drug undergoesdimerization in a $10 \mu \mathrm{M}$ DOX 50 solution.

The existence of dimers with two distinct geometries has been suggested by Agrawal et al. on the basis of the 2D NOESY NMR spectra of DOX in $\mathrm{D}_{2} \mathrm{O}$ and Molecular Dynamics (MD) calculations. ${ }^{27}$ In these two conformations, the arrangement of the 55 two DOX units would consist of the stacking of the aglycone moieties either in parallel or anti-parallel orientations, with the methoxy substituent of $\mathrm{D}$ ring pointing toward the exterior or the interior of the interplanar space, respectively. ${ }^{27}$ In the frame of the exciton theory, formation of such aggregates leads in principle to 60 a blue shift of the absorption spectrum with regards to that of the monomeric units. ${ }^{47}$ The important hypochromism at the absorption maximum and the concomitant hyperchromism in the low energy part of the absorption spectrum of dimerscould be explained by asignificant deviation from acollinear arrangement

65 of the transition dipole moments of the two DOX units. Indeed,MD calculations predicted significant angles between the two aromatic planes in the parallel and the antiparallel conformations,respectively. ${ }^{27}$ Additional indication forexcitonic couplingin DOX dimers has been found in the $\mathrm{CD}$ absorption 70 spectra of the drug, at concentrationsabove $10^{-5} \mathrm{M} \cdot{ }^{28}$ Theyexhibit a characteristic excitonic bisignate doublet in the $420-580 \mathrm{~nm}$ region, from which a $\sim 3000 \mathrm{~cm}^{-1}$ Davydov splitting can be estimated.

The fluorescence quantum yield of $10 \mathrm{mM}$ DOX solution for 75 excitation at $480 \mathrm{~nm}$ is known to be $3.9 \times 10^{-2} \cdot{ }^{28,}{ }^{33}$ Until now, DOX dimershave been considered as "non-fluorescent" species whose presencenoticeably reducesthe fluorescence intensity of the drug solutionwithout altering the shape of the fluorescence spectrum. ${ }^{27}$ Such a behavior provides evidence that the steady80 state fluorescence spectrum of DOX arises mainly from the monomers. At this point, it is noteworthy that, in contrast to the recent study of Rana et al., ${ }^{33}$ no effect of the excitation wavelength on the steady-state fluorescence spectrum of DOX in Tris buffer, can be observed. This, a priori, discards a possible 85 contribution of various ground-state species to the fluorescence signals.
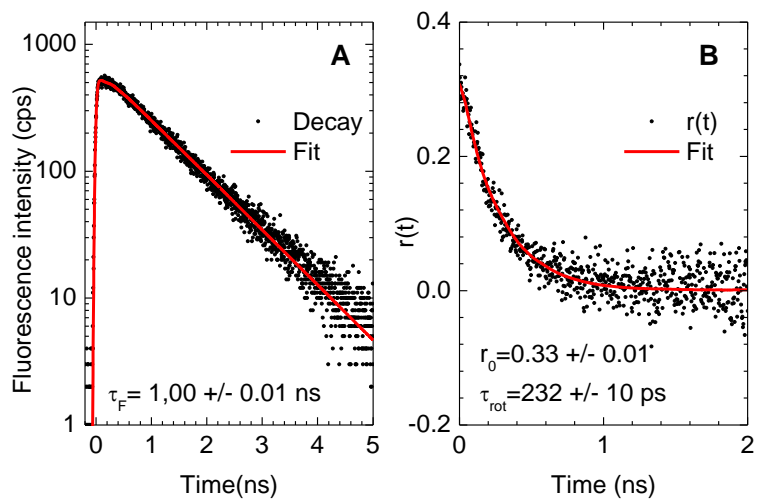

Fig. 3(A) Typical total fluorescence decay and (B)fluorescence anisotropy decay of $10 \mu \mathrm{M}$ DOX in Tris buffer, at $650 \mathrm{~nm}$. The red solid 
lines correspond to fits with mono-exponential functions convoluted with IRF. The fit of the anisotropy was extracted from a global fit of $I_{p a r}(t)$ and $\mathrm{I}_{\mathrm{perp}}(\mathrm{t})$.

\subsection{Nanosecond fluorescence decays and concentration 5 effects}

A typical total fluorescence decay of a $10 \mu \mathrm{M}$ DOX solution measured by TCSPC at $650 \mathrm{~nm}$ is shown in Fig. 3. DOX fluorescence exhibits a mono-exponential decay with a lifetime of $1 \mathrm{~ns}$, independently of the emission wavelength. There is only 10 weak variation of the fluorescence decays with the drug concentration. Slightly faster fluorescence decay (0.93 ns vs $1 \mathrm{~ns})$ has been found in a $450 \mu \mathrm{M}$ DOX solution, in which $88 \%$ of the drug molecules undergo dimerization.Theselifetimes arein good agreement with recent TCSPC measurements. ${ }^{28,}{ }^{32}$ The 15 exponential behaviour of DOX however markedly differs from some previous TCSPC measurements reporting biexponential fluorescence decays. ${ }^{24,}{ }^{29-33}$ Recently, the observation of biexponential decays has been correlated to the formation of some photodegradation products depending on the excitation 20 wavelength and intensity. ${ }^{32}$ In our case, the mono-exponential fluorescence decay of DOX in Tris buffer shows that, under our excitation conditions, the formation of photodegradation products can be excluded. It also precludes the above-mentioned contribution of several long-lived ground-state tautomers or 25 conformers to the fluorescence signals.

As illustrated in Fig. 3.B, the fluorescence anisotropy of a $10 \mu \mathrm{M}$ DOX solutionexhibits a fast exponential decay with a time constant of $232 \pm 10 \mathrm{ps}$ with $\mathrm{r}_{0}=0.33 \pm 0.02$. A similar decay has also been observed for a higher concentration $(450 \mu \mathrm{M})$ of DOX. 30 Using the Debye-Stokes-Einstein relationship, the rotational diffusion time is given by $\tau_{\text {rot }}=\eta \mathrm{V} / \mathrm{k}_{\mathrm{B}} \mathrm{T}$, where $\mathrm{V}, \mathrm{T}$ and $\eta$ are the hydrodynamic volume of DOX, the temperature and the viscosity of the solvent, respectively. With $\eta=1 \mathrm{cP}$, we estimate the effective volume of DOX to $938 \AA^{3}$. This value is fully 35 consistent with the volume of the anthracycline moiety. ${ }^{48}$ Since the same anisotropy decay is observedfor the concentrated solution of DOX, it provides strong evidence that, in the nanosecond regime, the fluorescence comes from the monomers solely.

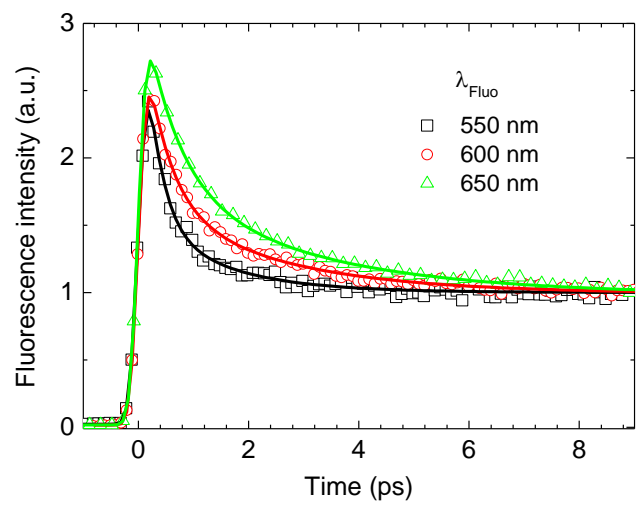

Fig. 4 Total fluorescence decays of $380 \mu \mathrm{M}$ DOX in Tris buffer,at 550, 600 and $650 \mathrm{~nm}$. The fits with a three-exponential function convoluted with a Gaussian representing the instrumental response function (FWHM $280 \mathrm{fs}$ ) are shown by solid lines.

\section{${ }_{45}$ 3.3. Ultrafast fluorescence decays: concentration effects}

In the femto-picosecond regime, the fluorescence of DOX in Tris buffer exhibits a more complex behavior. Kinetic traces recorded by FU at three wavelengths are represented in Fig. 4. Individual FU kinetic profiles were fitted using a three-exponential function 50 where the longest characteristic time was fixed to $1 \mathrm{~ns}$, in accordance with the fluorescence lifetime obtained from TCSPC. Results of the fits are gathered in Table 1.

Table 1. Time constants and amplitudes obtained from the individual fits of the fluorescence decays measured for $380 \mu \mathrm{M}$ DOX in Tris buffer.

\begin{tabular}{|c|c|c|c|}
\hline $\begin{array}{l}\text { DOX / Tris } \\
c=380 \mu M\end{array}$ & $\begin{array}{r}a_{1} \\
\tau_{1}\end{array}$ & $\begin{array}{r}a_{2} \\
\tau_{2} \\
\end{array}$ & $\begin{array}{l}a_{3} \\
\tau_{3} \\
\end{array}$ \\
\hline $550 \mathrm{~nm}$ & $\begin{array}{l}0.55 \pm 0.03 \\
271 \pm 20 \mathrm{fs}\end{array}$ & $\begin{array}{l}0.17 \pm 0.01 \\
1.4 \pm 0.2 \mathrm{ps}\end{array}$ & $\begin{array}{c}0.28 \pm 0.01 \\
1 \mathrm{~ns}\end{array}$ \\
\hline $600 \mathrm{~nm}$ & $\begin{array}{l}0.45 \pm 0.02 \\
440 \pm 35 \mathrm{fs}\end{array}$ & $\begin{array}{l}0.23 \pm 0.02 \\
2.2 \pm 0.1 \mathrm{ps}\end{array}$ & $\begin{array}{c}0.32 \pm 0.01 \\
1 \mathrm{~ns}\end{array}$ \\
\hline $650 \mathrm{~nm}$ & $\begin{array}{l}0.43 \pm 0.02 \\
607 \pm 46 \mathrm{fs}\end{array}$ & $\begin{array}{l}0.27 \pm 0.02 \\
2.8 \pm 0.1 \mathrm{ps}\end{array}$ & $\begin{array}{c}0.30 \pm 0.01 \\
1 \mathrm{~ns}\end{array}$ \\
\hline
\end{tabular}

55 Decays were fitted using a tree-exponential function where the longest time was fixed to $1 \mathrm{~ns}$.

As seen in Table 1, at all probed wavelengths, the fluorescence decays exhibits two fast components of a few hundred femtoseconds and about 2 ps respectively, followed bythe ${ }_{60}$ nanosecond component. The relative amplitudes $\left(\mathrm{a}_{1}\right.$ and $\left.\mathrm{a}_{2}\right)$ of the two fast time components exhibit a clear wavelength dependence concomitant with a significant increase of $\tau_{1}$ and $\tau_{2}$ with wavelength. The amplitudes of thesetwo fast components decrease dramatically with the drug concentration. In order to ${ }_{65}$ illustrate this effect, the normalized fluorescence decays measured at $600 \mathrm{~nm}$ for DOX solutions at four different concentrations are represented in Fig. 5. The result of the individual fits of the fluorescence decay traces measured at $600 \mathrm{~nm}$ is given in Table 2. For the highest concentrations, two 70 time-constants, one sub-ps and one ranging between 1-3 ps, are needed to reproduce the fast part of the FU fluorescence decays, whereas for the lowest concentration $(38 \mu \mathrm{M})$, only one picosecond time constant suffices. This provides evidence that the two fast components of the fluorescence decays of DOX are 75 correlated with the dimers.

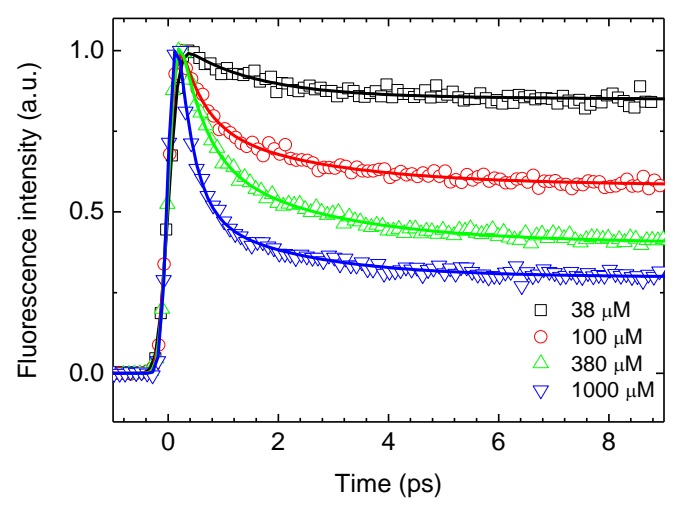

Fig. 5 Typical total fluorescence decays of DOX in Tris buffer, at $600 \mathrm{~nm}$ for different concentrations of the drug. The fit with a three-exponential 
function convoluted with a Gaussian representing the instrumental response function (FWHM $280 \mathrm{fs}$ ) are represented by the solid lines.

Table 2. Time constants and amplitudes obtained from the individual fits of the fluorescence decays measured for DOX in Tris buffer, at four 5 different concentrations.

\begin{tabular}{|c|c|c|c|}
\hline $\begin{array}{c}\text { DOX / Tris } \\
\lambda_{\text {Fluo }}=600 \mathrm{~nm}\end{array}$ & $\begin{array}{l}a_{1} \\
\tau_{1} \\
\end{array}$ & $\begin{array}{l}a_{2} \\
\tau_{2} \\
\end{array}$ & $\begin{array}{l}a_{3} \\
\tau_{3} \\
\end{array}$ \\
\hline $800 \mu \mathrm{M}$ & $0.50 \pm 0.02$ & $0.29 \pm 0.02$ & $0.21 \pm 0.01$ \\
\hline $9 \%$ mon. & $455 \pm 20 \mathrm{fs}$ & $2.1 \pm 0.1 \mathrm{ps}$ & $1 \mathrm{~ns}$ \\
\hline $380 \mu \mathrm{M}$ & $0.45 \pm 0.02$ & $0.23 \pm 0.01$ & $0.32 \pm 0.01$ \\
\hline $13 \%$ mon. & $441 \pm 35 \mathrm{fs}$ & $2.2 \pm 0.1 \mathrm{ps}$ & $1 \mathrm{~ns}$ \\
\hline $100 \mu \mathrm{M}$ & $0.35 \pm 0.10$ & $0.24 \pm 0.03$ & $0.41 \pm 0.01$ \\
\hline $23 \%$ mon. & $256 \pm 107 \mathrm{fs}$ & $1.9 \pm 0.3 \mathrm{ps}$ & $1 \mathrm{~ns}$ \\
\hline $38 \mu \mathrm{M}$ & - & $0.18 \pm 0.01$ & $0.82 \pm 0.01$ \\
\hline $35 \%$ mon. & - & $1.3 \pm 0.1 \mathrm{ps}$ & $1 \mathrm{~ns}$ \\
\hline
\end{tabular}

Decays were fitted using a tree-exponential function where the longest time was fixed to $1 \mathrm{~ns}$. The fraction of monomers for each concentration of the drug is given in the first column.

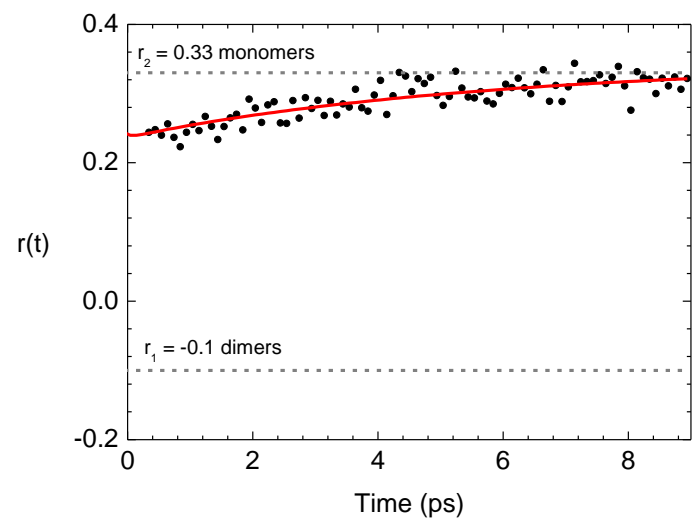

$10 \quad$ Fig. 6 Typical fluorescence anisotropy decay of $800 \mu \mathrm{M}$ DOX, at $600 \mathrm{~nm}$. The fit of the anisotropy represented by the red solid line has been extracted from the global fit of $I_{p a r}(t)$ and $I_{p e r p}(t)$. The dashed gray lines indicate the values of the anisotropy extracted from the fit $\left(\mathrm{r}_{1}=-0.10\right.$ \pm 0.02 and $\left.r_{2}=0.33 \pm 0.02\right)$.

15 Fig. 6 displays the typicalfluorescence anisotropy signal recorded on a time scale of $10 \mathrm{ps}$, at all the probed wavelengths. As shown in Fig. 6, the anisotropy traces exhibit a ca. 6 ps rise. This peculiar behaviour could be explained by the superposition of fluorescence stemming from two different species, namely the 20 dimers and the monomers. The short-lived fluorescence of the dimers is associated witha negative anisotropy $\left(r_{1}=-\right.$ $0.10 \pm 0.02)$, whereas the long-lived fluorescence of the monomers is associated with a positive anisotropy $\left(r_{2}=0.33 \pm 0.02\right)$, as observed in the TCSPC measurements. This 25 positive anisotropy remains constant over the whole observed time interval.

The fluorescence anisotropy depends on the natures of the absorbing and emitting species (states). The negative value of the dimer anisotropy indicates an important electronic relaxation, on 30 a time scale much faster than our time resolution ( $<200 \mathrm{fs})$. In other terms, the electronic transition associated with the dimer fluorescence is different from that associated to the photon absorption. The fluorescence anisotropy is given by:

$$
r(0)=\frac{1}{5}\left(3 \cos ^{2}(\theta)-1\right)
$$

where $\theta$ is the angle between the two transition dipoles. 35 Theanisotropy of -0.1 correspond toan angle of $66^{\circ}$.Conversely, the high anisotropy of the monomer fluorescence shows that,in this case, the excitation directly populates the emitting state.

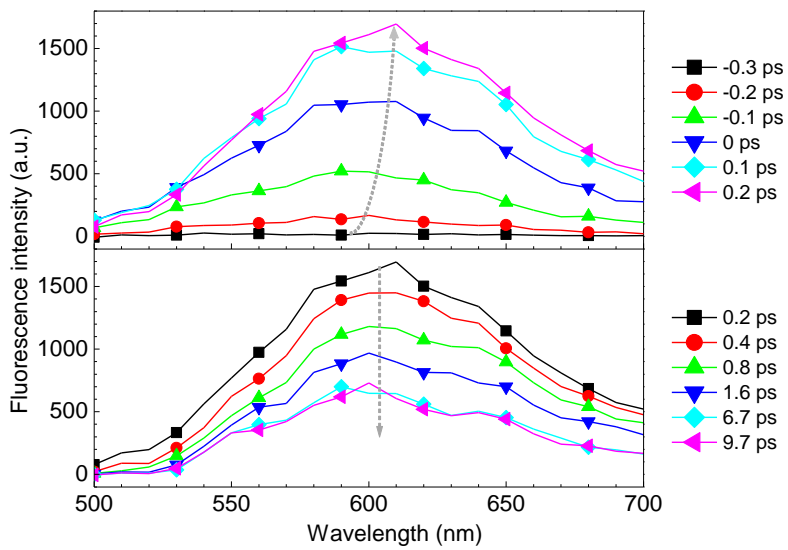

Fig. 7 Time-resolved fluorescence spectra of $380 \mu \mathrm{M}$ DOX in Tris buffer 40 between -0.3 and $0.2 \mathrm{ps}$ (upper panel) andbetween 0.2 and $9.7 \mathrm{ps}$ (lower panel).

\subsection{Origin of the ultrafast biexponential fluorescence decays of DOX dimers}

The short excited-state lifetime of the dimer fluorescence 45 indicates the existence of non-radiative processes that are not present in the monomers. The origin of the biexponential nature of the dimer fluorescence decays deserves further discussion. As shown in Table 1, the slight increase of the two short lifetimes with increasing wavelength suggests a dynamical red shift of the 50 fluorescence spectra of DOX on the femto-picosecond time scale. In order to confirm this hypothesis, the whole time-resolved spectra of DOX in Tris bufferat two different concentrationswere recorded. Fig. 7 illustrates the time evolutionof the spectra, over a time scale of $10 \mathrm{ps}$, at a DOX concentration of $380 \mu \mathrm{M}$. It can be 55 seen that the fluorescence spectrumof DOX exhibits a fast dynamical shift to the red within the duration of the apparatus function. Beyond a time delay of $0.2 \mathrm{ps}$, the fluorescence spectrum of DOX starts to decrease in intensity. After $6 \mathrm{ps}$, the fluorescence spectrum remains constant over the probed time 60 window.

The global kinetic analysis of the time-resolved fluorescence spectra of DOXrequired the sum of three exponential components. Fig. 8 displays the corresponding DAS.Their associated lifetimes are $0.22 \pm 0.02 \mathrm{ps}, 1.54 \pm 0.05 \mathrm{ps}$ for the ${ }_{65} 380 \mu \mathrm{M}$ DOX solution and $0.14 \pm 0.01 \mathrm{ps}, 1.45 \pm 0.06 \mathrm{ps}$ for the $100 \mu \mathrm{M}$ DOX solution, respectively. The longer time component associated to the monomer fluorescence has been fixed to $1 \mathrm{~ns}$, like for the individual fits. It is worth noting that this global analysis procedure, which implies a compartmental description of 70 the spectro-temporal evolution of the system, is not well-adapted if the fluorescence spectra display continuous time-dependent spectral shifts, which is the case in the present work. Still, thisprocedure is judged to provide a satisfactory qualitative description of the time-dependentfluorescence spectrum of 
DOX.Consequently, the global procedure can provide time constants thatdiffer slightly from those obtained by individual fits performed at given wavelengths.
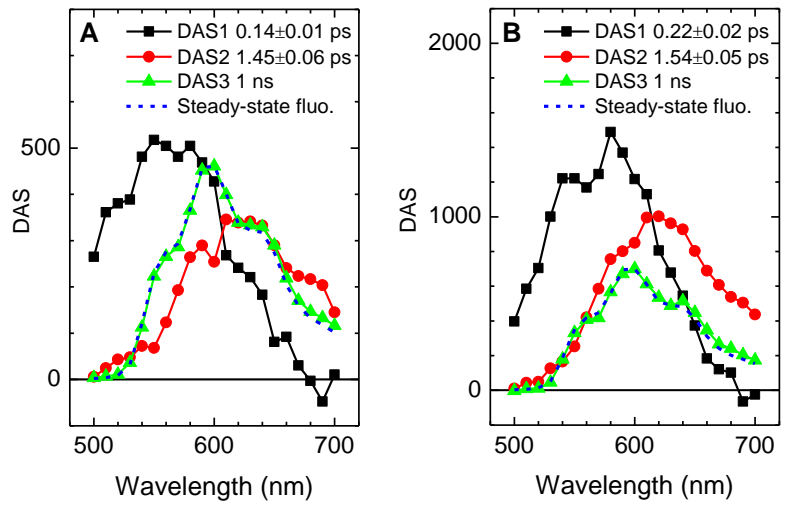

5 Fig. 8Decay-associated spectra DAS (solid lines) of the three time components found in the SVD analysis of the time-resolved fluorescence spectra of DOX in Tris buffer, at two different concentrations:

(A) $100 \mu \mathrm{M}$ and(B) $380 \mu \mathrm{M}$.For comparison, the steady-state fluorescence spectrum of DOX is repesented by the blue short-dashed lines.

10 As illustrated on Fig. 8, the relative amplitudes of the two first DAS with respect to DAS3 are clearly correlated to the drug concentration. Both of them can therefore be ascribed to the spectro-temporal changes of the dimer fluorescence spectrum. DAS1 (0.14 and $0.22 \mathrm{ps,} \mathrm{respectively)} \mathrm{displays} \mathrm{the} \mathrm{characteristic}$ 15 features of a fast dynamicalStokes shift of the fluorescence spectrum of dimers causing the continuous red shift of the band. The positive contribution between $500 \mathrm{~nm}$ and $650 \mathrm{~nm}$ in DAS1 arises from the fast initial decay on the blue side of thefluorescence band whereas the positive contribution above 650

$20 \mathrm{~nm}$ corresponds to the rise on the red side of the fluorescence band. DAS2 ( 1.5 ps) and DAS3 (1 ns)illustrate the fluorescence decay of the dimers and the monomers, respectively. The fluorescence spectrum ofthe dimers (DAS2) issignificantly redshifted with regards to that of the monomers(DAS3).At this point, 25 it is worth noting that the percentage of photons emitted by the dimers $\left(a_{2} \tau_{2} / \sum_{i=2}^{3} a_{i} \tau_{i}\right.$, see Table 1$)$ corresponds toless than $0.2 \%$ of the total fluorescence. The fluorescence quantum yield of the dimers is estimated to $\approx 10^{-5}$.

The fast dynamical Stokes shift of the dimer fluorescence in 30 Tris buffercan be described by an exponential function with a time component of $0.24 \pm 0.05$ ps (see SI Figure S1).Such a shiftcan be attributed to different processes occurring on the femto-picosecond time scale. First, a rationale may be offered by solvation dynamics, which is known to produce a spectrum like ${ }_{35}$ DAS1 with a negative amplitude in the red wing.For comparison, solvation dynamics of water has been shown to involve three components $(<1 \mathrm{ps})$, with an average time of about $0.5 \mathrm{ps}^{49-51}$ The dynamical shift of the DOX fluorescence, which is mostly described by DAS1 ( $0.2 \mathrm{ps})$, appears to be twice faster than the 40 average time of the solvation dynamics of water. This could be explained by the short lifetime of dimers preventing the observation of the entire solvation process. Besides the solvation dynamics, vibrational relaxation may also contribute to the earliest dynamics of the dimer fluorescence (DAS1). Such a
45 process would lead to a rapid narrowing of the emission band, in particular on the blue side, in agreement with the large amplitude decays observed around $550 \mathrm{~nm}$ (see Table 1).

The fluorescence spectrum of DOX dimers represented by DAS2 is found to be 25-nm red-shiftedwith respect to that of the 50 monomers. From the fraction of absorbing dimers vs.monomers, it can be estimated that the transition moment associated with the dimer fluorescence is about 5 times smaller than that of the monomer. The concomitant depolarization of the dimer fluorescence, observed immediately after excitation,is consistent 55 withthe excitationof anupper exciton statefollowed by a fast internal conversionto a lowerexciton statefrom whichemission arises. For this reason, the electronic transitions related to photon absorption and photon emission have different polarization.Note that the fast conversion between the two exciton states can also 60 be associated to important vibrational relaxation in the lower state, in line with the above-mentioned fast decay of the blue side of the dimer fluorescence.

Due to the weakertransition moment of the dimer fluorescence, one would expect its fluorescence lifetime to be longer than that 65 of the monomer.In contrast, the acceleration of the dimer fluorescence decay with respect to that of the monomer indicates the presence of additional non-radiative relaxation pathways.For instance, such fast excited-state decays have been reported inrhodamine 800 dimers. ${ }^{52}$ They were attributed to the 70 dissociation of the dimers in their excited-state leading to the formation of excited monomers. ${ }^{52}$ Such a process is not consistent with our experimental observationssincenodelayed rise of the monomers fluorescence is observed.Alternately, fast fluorescence decay in bichromophoric molecules such as perylene diimide ${ }^{53}$ 75 and naphthalene diimides ${ }^{54}$ have been assigned to a charge separationbetween the two units. In this context, we tentatively assign the fast fluorescence decay of DOXdimers to a charge separation process,occurringon a time scale of 1-2 ps, which is comparable to the diffusive part of the solvation dynamics. In 80 agreement with this attribution, the lifetime of DOX dimers in $\mathrm{D}_{2} \mathrm{O}$ is found to beslightly longer to that observed in Tris bufferand pure $\mathrm{H}_{2} \mathrm{O}$ (see SI Figures $\mathrm{S} 2$ and $\mathrm{S} 3$ andTables $\mathrm{S} 1$ and $\mathrm{S} 2$ ). Even though literature data on $\mathrm{D}_{2} \mathrm{O}$ solvation dynamics are scarce, the average solvation times of $\mathrm{D}_{2} \mathrm{O}$ islonger than that of ${ }_{85} \mathrm{H}_{2} \mathrm{O}{ }^{50,51}$

\section{Conclusion}

In this report, we have presented the first fluorescence study of DOX using a combination of time-resolved techniques with a time resolution up to $200 \mathrm{fs}$, in order to disentangle the various 90 molecular contributions to the fluorescence of the drug. Fluorescence decays of DOX in aqueous solution, at physiological conditions, were found to exhibit three exponential components whose amplitudes display a strong dependence on the drug concentration. The fluorescence lifetimes span from $950.2 \mathrm{ps}$ to $1 \mathrm{~ns}$. The fast components in the femto-picosecond regime can be unambiguously ascribed to the fluorescence of DOX dimers whereas the slowest component is due to the monomers.

In contrast to some previous time-resolved fluorescence 100 studies of DOX by TCSPC, we did not find any evidence of contributions of different tautomeric forms or photodegradation 
products. $^{24}$, 32-35 The global analysis of the time-resolved fluorescence spectra of DOX, measured at two different concentrationsin the femto-picosecond regime, allowed the extraction of the fluorescence spectra associated to monomers 5 and dimers. The fluorescence spectrum of monomers is similar to the steady-state fluorescence spectrum of DOX and has a lifetime of $1 \mathrm{~ns}$. In contrast, dimers display a significantly red-shifted and structureless fluorescence spectrum which undergoes a fast dynamic Stokes shift during a few hundred femtoseconds before 10 vanishing in about 2 ps.

The time-resolved fluorescence anisotropy decays provide evidence that the two species contribute to the fluorescence signals with distinct anisotropies, respectively. Dimer fluorescence is associated with a negative anisotropy of -0.1 15 while anisotropy of monomer fluorescence is equal to 0.33 . Such observation is consistent with the occurrence of a fast internal conversion between two excitonic states followed by a charge separation process leading to the fast decay of the dimer fluorescence. Comparative measurements in salt-rich aqueous 20 solution, pure water and heavy water suggest that this latter process could bepromoted bythe solvation dynamics.

\section{Acknowledgements}

The research leading to these results has received funding from COST MP0802 and from LASERLAB-EUROPE (grant 25 agreement no. 228334, EC's Seventh Framework Programme).

\section{Notes and references}

${ }^{a}$ Laboratoire Francis Perrin, CEA/DSM/IRAMIS/SPAM - CNRS URA 2453, 91191 Gif-sur-Yvette, France. Fax: + 33169088 707; Tel: +33169081 940; E-mail: pascale.changenet-barret@cea.fr

$30{ }^{b}$ Istituto per la SintesiOrganica e la Fotoreattività, CNR, via P. Gobetti 101, 40129 Bologna, Italy. Fax: +39051639 9844; Tel: +30 051639 9808; E-mail:ilse.manet@isof.cnr.it

$\dagger$ Electronic Supplementary Information (ESI) available: (i) Typical total fluorescence decay of DOX $(800 \mu \mathrm{M})$ in $\mathrm{D}_{2} \mathrm{O}$, (ii) Time constants and 35 amplitudes obtained from the individual fits of the fluorescence decays of DOX in $\mathrm{D}_{2} \mathrm{O}$, (iii) Typical total fluorescence decay of DOX $(800 \mu \mathrm{M})$ in $\mathrm{H}_{2} \mathrm{O}$,(iv) Time constants and amplitudes obtained from the individual fits of the fluorescence decays of DOX in $\mathrm{H}_{2} \mathrm{O}$.See DOI: 10.1039/b000000x/

\section{References}

40 1. D. Dal Ben, M. Palumbo, G. Zagotto, G. Capranico and S. Moro, Curr. Pharm. Des., 2007, 13, 2766-2780.

2. S. Moro, G. L. Beretta, D. D. Ben, J. Nitiss, M. Palumbo and G. Capranico, Biochem., 2004, 43, 7503-7513.

3. G. Capranico, F. Zunino, K. Kohn and Y. Pommier, Biochem., 1990, 45 29, 562-569.

4. C. Temperini, L. Messori, P. Orioli, C. D. Bugno, F. Animati and G. Ughetto, Nucleic Acids Res., 2003, 31, 1464-1469.

5. C. Frederick, L. Williams, G. Ughetto, G. Vandermarel, J. Vanboom, A. Rich and A. Wang, Biochem., 1990, 29, 2538-2549.

50 6. A. Thorburn and A. E. Frankel, Mol. Cancer Ther., 2006, 5, 197-199.

7. G. Minotti, P. Menna, E. Salvatorelli, G. Cairo and L. Gianni, Pharmacol. Rev., 2004, 56, 185-229.

8. G. L. Beretta and F. Zunino, in Anthracycline Chemistry and Biology II: Mode of Action, Clinical Aspects and New Drugs, ed. SpringlerVerlag, Berlin2008, pp. 1-19.

9. F. Arcamone, F. Animati, G. Capranico, P. Lombardi, S. Pratesi, S. Manzini, R. Supino and F. Zunino, Pharmacol. Ther., 1997, 76, 117124.

10. M. Dalmark and H. H. Storm, J. Gen. Physiol., 1981, 78, 349-364.
60 11. B. Cerroni, E. Chiessi, S. Margheritelli, L. Oddo and G. Paradossi, Biomacromolecules, 2011, 12, 593-601.

12. P. Horcajada, T. Chalati, C. Serre, B. Gillet, C. Sebrie, T. Baati, J. F. Eubank, D. Heurtaux, P. Clayette, C. Kreuz, J. S. Chang, Y. K. Hwang, V. Marsaud, P. N. Bories, L. Cynober, S. Gil, G. Ferey, P.

65 Couvreur and R. Gref, Nat. Mater., 2010, 9, 172-178.

13. H. Huang, Q. Yuan, J. S. Shah and R. D. K. Misra, Adv. Drug Deliv. Rev., 2011, 63, 1332-1339.

14. C. T. Huynh, M. K. Nguyen, J. H. Kim, S. W. Kang and B. S. Kim, Soft Matter, 2011, 7, 4974-4982.

70 15. A. Lowery, H. Onishko, D. E. Hallahan and Z. Z. Han, J. Controlled Release, 2011, 150, 117-124.

16. D. M. Ren, F. Kratz and S. W. Wang, Small, 2011, 7, 1051-1060.

17. F. Wang, Y. C. Wang, S. Dou, M. H. Xiong, T. M. Sun and J. Wang, ACS Nano, 2011, 5, 3679-3692.

75 18. Y. Yan, A. P. R. Johnston, S. J. Dodds, M. M. J. Kamphuis, C. Ferguson, R. G. Parton, E. C. Nice, J. K. Heath and F. Caruso, ACS Nano, 2010, 4, 2928-2936.

19. R. Anand, F. Manoli, I. Manet, S. Daoud-Mahammed, V. Agostoni, R. Gref and S. Monti, Photochem. Photobiol. Sci., 2012, 11, 12851292.

20. M. Menozzi, L. Valentini, E. Vannini and F. Arcamone, J. Pharm. Sci., 1984, 73, 766-770.

21. L. Angeloni, G. Smulevich and M. P. Marzocchi, Spectrochim. Acta, Pt. A: Mol. Spectrosc., 1982, 38, 213-217.

85 22. L. Gallois, M. Fiallo and A. Garnier-Suillerot, Biochim. Biophys. Acta, 1998, 1370, 31.

23. K. K. Karukstis, E. H. Z. Thompson, J. A. Whiles and R. J. Rosenfeld, Biophys. Chem., 1998, 73, 249-263.

24. T. Htun, J. Fluores., 2004, 14, 217-222.

90 25. M. P. Evstigneev, V. V. Khomich and D. B. Davies, Russ. J. Phys. Chem., 2006, 80, 741-746.

26. I. J. McLennan, R. E. Lenkinski and Y. Yanuka, Can. J. Chem., 1985, 63, 1233-1238.

27. P. Agrawal, S. K. Barthwal and R. Barthwal, Eur. J. Med. Chem., $95 \quad 2009,44,1437-1451$.

28. R. Anand, S. Ottani, F. Manoli, I. Manet and S. Monti, RSC $A d v$., 2012, 2, 2346-2357.

29. N. Husain, R. A. Agaria and I. M. Warner, J. Phys. Chem. A, 1993, 97, 10857-10861.

100 30. R. Goldman, T. Facchinetti, D. Bach, A. Raz and M. Shinitzky, Biochim. Biophys. Acta, 1978, 512, 254-269.

31. T. G. Burke, M. Israel, R. Seshadri and J. H. Doroshow, Biochim Biophys. Acta, 1989, 982, 123-130.

32. K. Nawara, P. Krysinski and G. J. Blanchard, J. Phys. Chem. A, 2012, 116, 4330-4337.

33. D. K. Rana, S. Dhar, A. Sarkar and S. C. Bhattacharya, J. Phys. Chem. A, 2011, 115, 9169-9179.

34. M. Fiallo, A. Laigle, M. N. Borrel and A. Garnier-Suillerot, Biochem., 1993, 45, 659-665.

110 35. O. Hovorka, V. Subr, D. Vetvicka, L. Kovar, J. Strohalm, M. Strohalm, A. Benda, M. Hf, K. Ulbrich and B. Rihova, Eur. J. Pharm. Biopharm., 2012, 76, 514-524.

36. T. Gustavsson, A. Sharonov and D. Markovitsi, Chem. Phys. Lett., 2002, 351, 195.

115 37. D. Markovitsi, D. Onidas, F. Talbot, S. Marguet, T. Gustavsson and E. Lazzarotto, J. Photochem. Photobiol. A: Chem., 2006, 183, 1-8.

38. T. Gustavsson, G. Baldacchino, J.-C. Mialocq and S. Pommeret, Chem. Phys. Lett., 1995, 236, 587-594.

39. E. R. Henry and J. Hofrichter, Methods Enzymol., 1992, 210, 129.

120 40. P. Changenet-Barret, P. Plaza, M. M. Martin, H. Chosrowjan, S. Taniguchi, N. Mataga, Y. Imamoto and M. Kataoka, J. Phys. Chem. C, 2009, 113, 11605-11613.

41. D. K. Palit, H. Pal, T. Mukkherjee and J. Mittal, J. Chem. Soc. Farad. Trans., 1990, 86, 3861-3869.

125 42. M. M. L. Fiallo, H. Tayeb, A. Suarato and J. P. S. A. GarnierSuillerot, 1998, 87, 967-975., J. Pharm. Sci., 1998, 87, 967-975.

43. G. Smulevich, L. Angeloni, S. Giovannardi and M. P. Marzocchi, Chem. Phys., 1982, 65, 313-322.

44. R. Kiraly and R. B. Martin, Inorganica Chimica Acta-Bioinorganic Chemistry, 1982, 67, 13-18. 
45. L. Messori, C. Temperini, F. Piccioli, F. Animati, C. D. Bugno and P. Orioli, Biorg. Med. Chem., 2001, 9, 1815-1825.

46. J. B. Chaires, N. Dattagupta and D. M. Crothers, Biochem., 1982, 21, 3927-3932.

5 47. M. Kasha, H. R. Rawls and M. Ashraf El-Bayoumi, Pure Appl. Chem., 1965, 11, 371-392.

48. J. P. Rasimas and G. J. Blanchard, J. Phys. Chem., 1995, $9911333-$ 11338.

49. R. Jimenez, G. R. Fleming, P. V. Kumar and M. Maroncelli, Nature, 1994, 369, 471-473.

50. D. Pant and N. E. Levinger, J. Phys. Chem., 1999, 103, 7846-7852.

51. M. Sajadi, M. Weinberger, H.-A. Wagenknecht and N. P. Ernsting, Phys. Chem. Chem. Phys., 2011, 13, 17768-17774.

52. K. Sekiguchi, S. Yamaguchi and T. Tahara, J. Phys. Chem. A, 2006, 15 110, 2601-2606.

53. J. M. Giaimo, A. V. Gusev and M. R. Wasielewski, J. Am. Chem. Soc., 2002, 124, 8530-8531.

54. N. Banerji, A. Fürstenberg, S. Bhosale, A. L. Sisson, N. Sakai, S. Matile and E. Vauthey, J. Phys. Chem. B, 2008, 112, 8912-8922.

20 